\title{
Kinetic viscoelasticity during early polymer-polymer spinodal dewetting
}

\author{
J. Lal $\odot,{ }^{1,2,3}$ L. B. Lurio, ${ }^{3}$ D. Liang, ${ }^{2}$ S. Narayanan, ${ }^{4}$ S. B. Darling $\odot,{ }^{5}$ and M. Sutton $\oplus^{6}$ \\ ${ }^{1}$ Materials Science Division, Argonne National Laboratory, Argonne, Illinois 60439, USA \\ ${ }^{2}$ Intense Pulsed Neutron Source, Argonne National Laboratory, Argonne, Illinois 60439, USA \\ ${ }^{3}$ Department of Physics, Northern Illinois University, DeKalb, Illinois 60115, USA \\ ${ }^{4} X$-ray Science Division, Argonne National Laboratory, Argonne, Illinois 60439, USA \\ ${ }^{5}$ Chemical Sciences and Engineering, Argonne National Laboratory, Argonne, Illinois 60439, USA \\ ${ }^{6}$ Physics Department, McGill University, Montréal, Canada H3A 2T8
}

(Received 26 May 2021; accepted 29 November 2021; published 8 December 2021; corrected 5 January 2022)

\begin{abstract}
The dewetting kinetics of a supported polymer bilayer were measured in situ using coherent grazing-incidence $\mathrm{x}$-ray scattering. X-ray photon correlation spectroscopy provides both the two-time correlation functions and the cross-correlation function which measures the average spatial shift of the speckles produced by the coherent $\mathrm{X}$ rays. The stress in the ultrathin top dewetting film can be directly observed due to the exquisite sensitivity to sample curvature changes provided by the $\mathrm{x}$-ray speckle correlation functions. The hole-opening events in the film are found to be associated with significant changes to the stress. These results are interpreted through an analogy between viscoelastic spinodal dewetting and early-stage bulk viscoelastic phase separation. The frequency of hole-initiation events during dewetting decreases with time as a power law, and the power-law exponent can be linked to nonlinear viscoelastic effects, showing similarity in their stress relief dynamics to aftershock decays.
\end{abstract}

DOI: 10.1103/PhysRevResearch.3.043162

\section{INTRODUCTION}

The kinetics of hole opening during dewetting displays a fascinating interplay of viscous and elastic energies. An improved understanding of these processes not only promises to advance knowledge of important aspects of polymer physics, and the technology of polymer film adhesion, but also may shed light on a broad range of physical systems where elastic and viscous forces have comparable magnitudes. Measurement of the dewetting velocity of large isolated holes due to nucleation in viscoelastic polymer thin films has already been used to get access to mechanical properties of polymers [1-6]. These experiments point out the importance of viscoelasticity, not just viscosity, in understanding the dewetting process in thin polymer films on a solid substrate. Less is known, however, about dynamics when dewetting rupture is reached at roughly the same time for the whole film as in spinodal dewetting rather than from isolated nucleating holes. In our previous experimental studies of late-stage coarsening, a close analogy between spinodal dewetting and normal bulk phase separation kinetics [7] was observed. In this paper on the early stages of viscoelastic spinodal dewetting, many similarities to bulk phase separation, called viscoelastic phase separation [8-11], are shown.

Published by the American Physical Society under the terms of the Creative Commons Attribution 4.0 International license. Further distribution of this work must maintain attribution to the author(s) and the published article's title, journal citation, and DOI.
While dewetting of molten polymer thin films on solid substrates has been widely studied since the seminal work by Reiter [12,13], the hole formation and early coarsening of spinodally initiated dewetting in molten polymer films on molten polymer substrates represent a more complex situation. The bilayer studied here is unstable, and, if heated above the glass transition, the top surface dewets from the underlayer. Here, the mode that leads to dewetting is a socalled peristaltic mode $[14,15]$, as shown schematically in Fig. 1(a), where the displacements of the free surface and the polymer-polymer interface are in antiphase. The amplitude of displacement of the surface is much smaller than the displacement at the buried polymer-polymer interface of the bilayer as the displacement amplitude is inversely proportional to their respective surface and effective interface tensions. The effective interface tension is an order of magnitude smaller $\left(2.1 \times 10^{-3} \mathrm{~N} / \mathrm{m}\right)$ at the buried interface compared with the surface tension of the top layer $\left(33.7 \times 10^{-3} \mathrm{~N} / \mathrm{m}\right)$ [16]. Otherwise, the same condition for marginal stability applies as for single layers on solid substrates [7,14,15]. Furthermore, experimentally it was found that these strongly immiscible films have a thin interface $(\sim 25 \AA)$ where polymers intermix mostly on the segmental scale. The interdiffusion is small $[17,18]$. The effect of the fluidity of the underlayer is to create a slip boundary condition for the top layer [18] since the slip length at the interface exceeds the thickness ( $h=180 \AA$ ) of the top layer $[16,19,20]$. The bulk dynamics of the underlayer do not play a significant role due to its high viscosity, but instead this layer acts like a slippery, solid substrate [14-16,21]. If the van der Waals attraction of the top polymer layer for itself is sufficiently stronger than the attraction towards the 

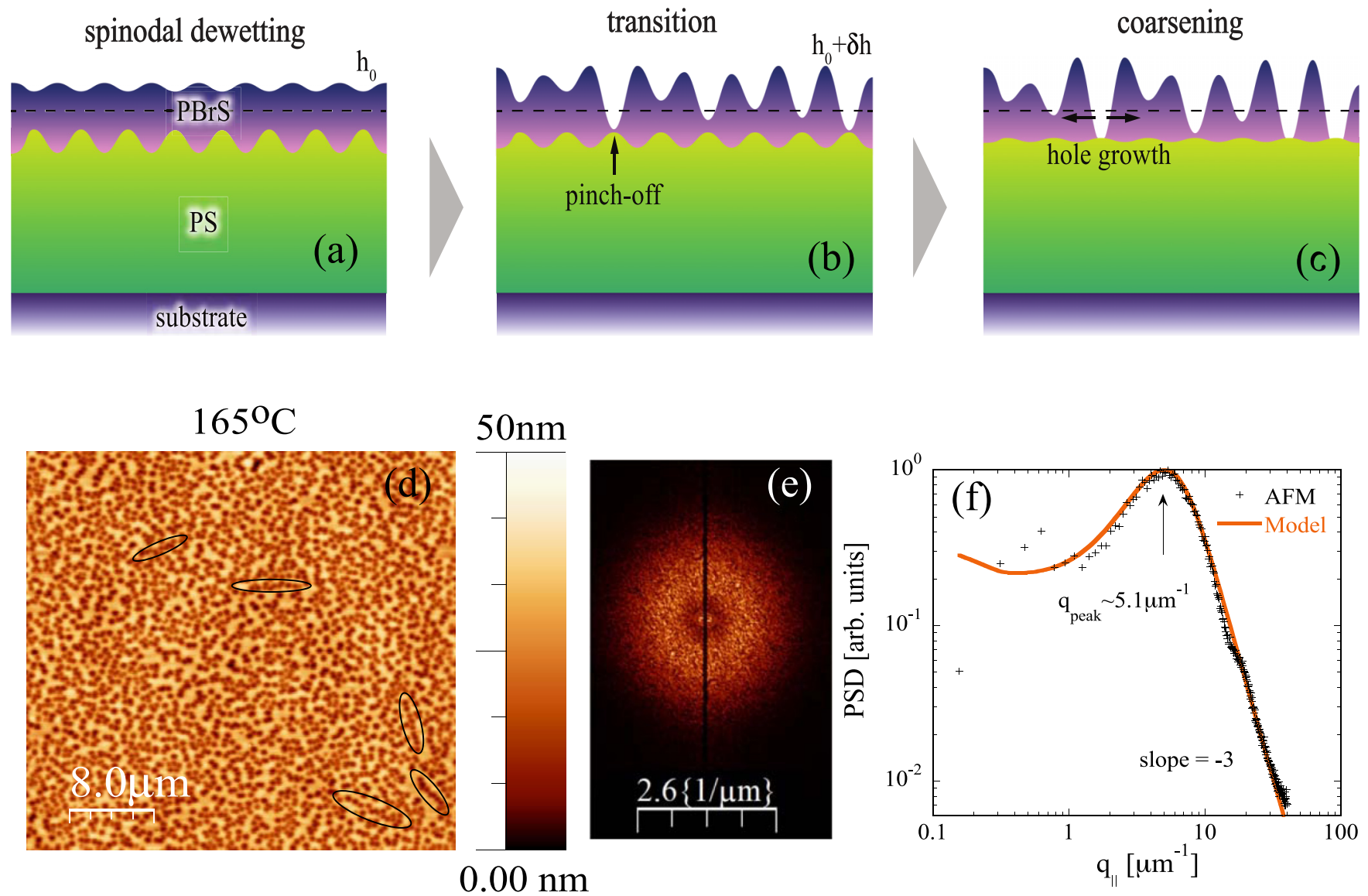

FIG. 1. Schematic snapshots of the time evolution of a two-layer PBrS-PS film on a Si substrate with slip at the PBrS-PS interface. (a) Idealized schematic of the "peristaltic mode" leading to dewetting, where the displacements of the free surface and the polymer-polymer interface are in antiphase $[14,15]$. For the case of a dewetting system, the long-range van der Waals forces become dominant at small thickness, and then the film is unstable: The peristaltic mode is amplified for a characteristic length scale [14]. The film ruptures in the thinner parts, leading to hole formation. (b) At the film rupture point, the hole depth becomes comparable to the top film thickness $h$. AFM measurements confirmed that the average depth of the holes is equal on average to the top layer thickness [16]. This kinetics is followed in situ by the XPCS experiments reported in this paper. (c) The late-stage coarsening of the film. The idealized schematic is further affected by viscoelastic effects. (d) At the end of the XPCS measurement the AFM measurements were performed (Supplemental Table S1 [16]). Highlighted in black are a few arrays of nucleated holes; such a process of nucleating holes in arrays is also observed in simulations and experiments of viscoelastic phase separation in polymers [38]. (e) Fast Fourier transform (FFT) of the AFM image in (d). (f) The power spectral density (PSD) of the AFM image. The solid line is a calculation from capillary waves and island (holes) model $[39,40]$. Details of the model are explained in the Supplemental Material [16].

polymer underlayer, and if the underlayer is sufficiently thick, then the top layer will be unstable to breakup and thickening. Depending on the specific values of the Hamaker constants and thickness of the underlayer and top layer, this breakup can occur through nucleation or spinodally. A calculation based on previous work $[22,23]$ shows that for the current system the breakup should be spinodal [16,23-26] with an expected critical wavelength $[15,23,27,28]$. In addition, the top layer is expected to be viscoelastic as shown in previous work $[4,18]$.

There are several attempts theoretically to describe "single" hole-initiation (pinch-off) events on a substrate by modeling [29-33]. These generally describe self-similar dynamics and finite time singularities that arise during thinning and rupture of films. Based on them, hole formation in the film can be separated into two distinct and consecutive steps reflected by power laws: (1) the thinning of the top layer at the troughs until the top layer initiates holes and (2) a regime of prolonged periods of hole growth or expansion. The two steps are governed by different flow mechanisms with surface tension forces having different roles [29-33]. The flow dynamics that lead to the layer hole initiation are driven by van der Waals attraction and resisted by surface and viscoelastic forces. The growth of a hole is driven by surface tension forces and hindered by viscoelastic forces.

Dewetting at the top interface begins via capillary wave fluctuations [27,34] [Fig. 1(a)]. Once their amplitude becomes comparable to the film thickness, rupturing (or pinch-off [35-37]) starts along the troughs of the capillary waves leading to the opening of holes in the plane of the still-connected top film [Fig. 1(b)]. Further dewetting of the holes in the top film proceeds via slow coarsening [Fig. 1(c)]. The transition stage where holes are initiated and start to grow [Fig. 1(b)] is the focus of this paper. 
The surface capillary wave undulations (height fluctuations) give rise to a pressure gradient which drives the film instability if the effective Hamaker constant is negative (dewetting liquid). In the normal model of the kinetics of this process, one would expect the spinodal dewetting dynamics to be determined by a balance between the driving force due to the van der Waals potential gradient and the rate of self-diffusion of the polymer. However, this model ignores viscoelastic stress. The relation between the length scale due to the order parameter fluctuations (height fluctuations [7]) and the viscoelastic length scale of the polymer determines how the kinetics of dewetting proceeds in the early stages of multiple hole formation in the film [Figs. 1(a) and 1(b)]. The viscoelastic length is the characteristic length scale above which dynamics are dominated by diffusion and below which dynamics are dominated by viscoelastic effects [41]. This length is a rheological length scale (many times the radius of gyration as shown in the Supplemental Material [16]) intrinsic to entangled polymers $[42,43]$. It is known that the bulk stress suppresses the growth of the composition fluctuations and the diffusion [44] in bulk viscoelastic phase separation [8-11]. Viscoelastic dewetting kinetics will be shown to be analogous to early viscoelastic bulk phase separation kinetics below.

Previous experimental and theoretical work on single hole opening in suspended $[2,45]$ and ultrathin unstable supported polymer films [46] with slip at the interface gives similar behavior. When the radius of the single hole opening is less than $R_{c}=\sqrt{h b}$, where $h$ is the film thickness and $b$ is the slip length [16], the supported viscoelastic films, as with suspended films, show no evidence of a rim collecting the liquid from the growing hole. Instead, there is elastic stress generated at the hole which leads to long-range plug flows, related to a uniform small thickening of the film. Another study of the very early stage of the dewetting of multiple holes shows that this process does not depend on the molecular weight of the polymer [1], further indicating that the driving force is elastic.

The dominance of elastic forces in dewetting at short times has been predicted [45] for thin polymer films. This hypothesis [1] is supported by the observation that the acting stress $\sigma$ due to capillary forces at the periphery of the holes, which is equal to the spreading parameter $|S|$ divided by $h$ (i.e., $|S| / h$ ), is comparable to the plateau modulus, $G$, of the polymer layer. For the top polymer layer in this paper, these parameters are $|S|=\left|-5.4 \times 10^{-3}\right| \mathrm{N} / \mathrm{m}, h=180 \AA$, and $G_{\text {top-layer }}=$ $1.8 \times 10^{5} \mathrm{~N} / \mathrm{m}^{2}[16,17,47]$ : Thus it is similarly expected that the capillary driving forces will be sufficiently strong to elastically deform the periphery of the opening holes, as $|S| / h$ $\left(\sim 3 \times 10^{5} \mathrm{~N} / \mathrm{m}^{2}\right)$ is of the same order of magnitude as $G$.

In the regime of very early spinodal dewetting, multiple correlated fast hole-initiation events (called pinch-offs during droplet formation in phase separation) lead to hole formation. Stress at hole initiation or pinch-off involves highly nonlinear processes (chain extension, pull out, and retraction) and cannot be accurately calculated theoretically but only provided as an experimental input. Multiple hole formation in a short interval of time makes this process more complex than single hole formation. The kinetics are best explained by analogy to viscoelastic phase separation [8-11]. As holes form, the flow of material from holes to the rest of the film happens faster than the stress generated can relax. Thus elastic deformation of the polymer film due to hole formation and growth is spontaneously generated during the dewetting process itself. The stress from this process compresses the connected polymer film where the polymer chains can store the bulk stress. As a result, the initial growth of height fluctuations, which is characteristic of usual spinodal dewetting, is significantly suppressed. Based on the spring interaction model $[38,44]$ the viscoelastic stress overwhelms the interfacial tension in viscoelastic phase separation.

In the absence of elastic energy, hole opening is energetically favorable since it minimizes the disjoining (van der Waals) energy. When the polymer is elastically stressed, there is an additional energy term associated with opening a hole due to the compression of the remaining polymer film. The energy for opening a new hole is even higher when there is existing stress from the last round of hole openings. Thus a viscoelastic polymer film can show periodic behavior in hole openings. A round of hole opening is followed by a delay required for the stress to relax, followed by another round of hole opening. In the absence of viscoelasticity, holes would open continuously. As holes grow, the film thickens, which also decreases the dewetting driving force [16], lengthening the time between hole-opening events.

The energetics of hole opening was modeled as follows. Consider a single hole of area $a_{H}$. Since the film is unstable to dewetting, a negative disjoining energy per unit area [16], $-\phi$, was assumed. The opening of a hole of area $a_{H}$ then decreases the film energy by $-\phi a_{H}$. In the absence of stress relaxation, the opening of a hole also contributes to an increase in the film energy due to increased stress in the film. The stress energy is approximately given by $E_{S}=\frac{1}{2} \kappa h \frac{a_{H}^{2}}{A}$ [16]. Here, $\kappa$ is a modulus proportional to the bulk modulus, but not necessarily equal depending on how the film reacts to compression, $h$ is the film thickness, and $A$ is the total polymer area over which this film compression spreads (not necessarily the entire film area). In this simplified model, a hole will continue to open until $E_{d}+E_{s}=0$ or $a_{H}=\frac{2 A \phi}{\kappa h}$. As the stress relaxes, the hole size will slowly increase. If a minimum nucleation size for a hole to open, $a_{0}$, is assumed, then holes will not form if $a_{0}>\frac{2 A \phi}{\kappa d}$. However, since holes do form, it is supposed that this condition is not met.

Now it is assumed that a series of holes have already formed resulting in an unrelaxed stress $\sigma$. In this case, new holes will not open until the unrelaxed stress that creates a barrier to new holes opening is reduced below $\frac{\phi}{h}+\frac{1}{2} \kappa \frac{a_{0}}{A}$ [Eq. (2)].

$$
h \sigma<\phi+\frac{1}{2} \kappa h \frac{a_{0}}{A} .
$$

The stress is modeled as an exponential decay, $\sigma=\sigma_{0} e^{-t / \tau}$ (though it is much more complicated as time-dependent viscosity effects are neglected in this simple model); then the condition for the time delay between hole-opening events, $\Delta t$, is given by

$$
\sigma_{0} e^{-\Delta t / \tau}=\frac{\phi}{h}+\frac{1}{2} \kappa \frac{a_{0}}{A} .
$$


A key feature of viscoelastic phase separation is a coupling between concentration, velocity, and stress fields which occurs when one phase exhibits dynamical properties that are significantly different from those of the other. This is referred to as "dynamic asymmetry" [8-11]. Here, the asymmetry is between the regions of polymer film and the regions occupied by holes. This stage of spinodal dewetting shows analogy to the viscoelastic phase separation [48] due to the dynamic asymmetry between holes and polymer film (which supports all the stress) $[41,49]$.

An important consequence of viscoelastic phase separation is order parameter switching, which has been described as an interchange between the composition (here, height fluctuations) and the deformation tensor, in viscoelastic phase separation [9]. Here, one order parameter, mechanical deformation, is dominant during formation of the holes, and the other order parameter is dominant when the growth of holes is observed. Initially, alternating episodes of nucleation and growth are observed until the growth of holes becomes dominant.

In viscoelastic dewetting, the constitutive equation specific to the material is needed to describe viscoelasticity theoretically. This material-specific feature is the origin of the intrinsically nonuniversal nature of viscoelastic dewetting. Viscoelastic effects in polymers are kinetic effects; they cannot be included in the Hamiltonian, and dynamic equations are required to describe them. It is quite difficult to make any theoretical prediction, such as on the exact form of stress generated during viscoelastic dewetting, as there are no theories for the rheological behavior of a system in nonequilibrium states $[41,49]$. Here, the coherent $\mathrm{x}$-ray speckle is used to probe this kinetic viscoelasticity during dewetting.

At longer times due to the onset of flow, hole growth, rather than opening, starts to predominate, which signals the beginning of the coarsening stage. If the coarsening rate is determined by diffusion, the dynamics should be universal. In the present case, where the rate of viscoelastic relaxation is sufficiently slow (e.g., when the reptation time of the polymer $T_{\text {rep }}$ is slower than the fast deformation time due to hole formation), the coarsening is determined by the material-specific viscoelasticity and is not universal.

The dewetting process was quantified through three distinct measures. First, the self-similar evolution of the average dewetting structure is measured through the time evolution of the average surface diffuse scattering, $\left\langle I\left(q_{\|}\right)\right\rangle$, where $q_{\|}$ is defined as the in-plane component of the wave vector; this is like conventional grazing-incidence small-angle X-ray scattering (GISAXS). Secondly, the time evolution of fluctuations about the average was quantified via measurement of the two-time correlation function, which can be obtained by time correlation of the speckle pattern resulting when the sample is illuminated using a coherent incident x-ray beam [50]. Thirdly, the stress in the upper film as dewetting holes form during the process was measured through subpixel speckle shifts. These shifts were caused by film stress-induced curvature of the supporting silicon substrate.

\section{MATERIALS AND METHODS}

The system studied is a poly(4-bromostyrene) ( $\mathrm{PBrS})$ thin film deposited on an immiscible and nonwettable polystyrene
(PS) sublayer on a silicon substrate. Monodisperse PS samples (molecular weight $M_{w}=1500 \mathrm{kDa}$ ) from Pressure Chemical were used. The thickness of the PS bottom layer was fixed to $1000 \AA$ and was deposited on precleaned $15 \times 15 \times 0.5-\mathrm{mm}$ silicon wafers. The $\operatorname{PBrS}\left(M_{w}=350 \mathrm{kDa}\right.$; the fraction of brominated monomers was 0.89) was used as the top layer. The bilayers were prepared by spin-coating from toluene solution PBrS layers of thickness $\sim 180 \AA$ onto a glass slide. This thin layer was then floated from deionized water onto the $1000 \AA$ PS sublayer on the silicon substrate. The bilayer film was annealed at $90{ }^{\circ} \mathrm{C}$ (to reduce stress but not start dewetting) for $12 \mathrm{~h}$. This temperature is below the glass transition temperature of PS $\left(T_{g}=100^{\circ} \mathrm{C}\right)$ and $\mathrm{PBrS}$ (100\% brominated $\left.\mathrm{PBrS}, T_{g}=142^{\circ} \mathrm{C}[16,47,51]\right)$. There was additional annealing of the top $\mathrm{PBrS}$ layer (reptation time $T_{\text {rep }}=33 \mathrm{~s}$ [16]) prior to the observation window of the kinetics (the $t_{w}=978 \mathrm{~s}$ after quenching to $165^{\circ} \mathrm{C}$ ). Since there is a slip boundary condition that causes less stress at the buried interface, the dewetting dynamics will represent the properties of the equilibrated sample [16]. Furthermore, based on the root-mean-square roughness obtained from the measured reflectivity profiles at room temperature [16], no effect of the conformal roughness of the substrate on high-temperature dewetting [52] is expected.

Grazing-incidence $\mathrm{x}$-ray photon correlation spectroscopy (XPCS) experiments were performed at the x-ray energy of $7.34 \mathrm{keV}$ with beam size $20 \times 20 \mu \mathrm{m}$ at the sector 8 -ID-I beamline at the Advanced Photon Source, Argonne National Laboratory, as described previously [4]. The distance from sample to CCD camera detector was $3.76 \mathrm{~m}$. The kinetics of dewetting was followed in situ via diffuse surface $\mathrm{X}$-ray scattering, recorded by successive detector frames. The exposure time was $0.06 \mathrm{~s}$ per frame, and the time interval between frames was $1.99 \mathrm{~s}$. Partially coherent $\mathrm{x}$ rays are incident at $0.146^{\circ}$, which is below the critical angle for total external reflection of both layers. The electric field intensity calculation shows that no field penetrates the bilayer at this incident angle and just the top surface is probed (Supplemental Fig. S12 [16]). X-ray scattering measurements were performed, with the samples placed in a temperature-controlled vacuum chamber $\left(\sim 10^{-4}\right.$ torr). Dewetting of $\mathrm{PBrS}$ thin films above $T_{g}$ of both layers is followed in situ using XPCS.

Figure 1(d) shows an atomic force microscopy (AFM) image of the surface topography of the bilayer at the end of the experiments (after $72 \mathrm{~min}$ ). It reveals interesting characteristics of pattern formation, i.e., one-dimensional arrays of holes (some highlighted with black ovals). Such one-dimensional arrays of droplets have been observed in simulations (i.e., holes here) [38] and in experiments of viscoelastic phase separation in polymers [10]. This reveals the spatial elastic coupling during hole formation. If two holes are nucleated or formed close to each other at about the same time, the deformation field around them, which is induced by a slight contraction of the polymer matrix, becomes anisotropic. It is conjectured that the tensile stress is concentrated at the edges of the array of holes, while the compressive stress is concentrated normal to the array. This helps in the creation of new holes at the edges and thus leads to a further increase in the number of holes in the different arrays in a correlated manner. This elastic coupling between emerging holes is a 
characteristic of mechanically dominated pattern evolution [38] in the early stages. When diffusion sets in, hole formation in arrays gets disrupted (as discussed above). These processes were followed in situ as reported below.

Figure 1(e) is the fast Fourier transform (FFT) of the AFM image in Fig. 1(d). Figure 1(f) is the power spectral density (PSD) of the AFM image. PSD is the Fourier transform of the height-height correlation function $[16,40]$. The measured PSD shows a peak at $5.1 \mu \mathrm{m}^{-1}$, which is formed by the regular mesoscopic structures of the holes. The solid line shows the fitted PSD from a model of capillary waves and islands (holes in our case) as proposed previously [39,40], and details of the model are shown in the Supplemental Material. The model describes the measured PSD well with the right asymptotic slope for $q_{\|}^{-3}$ as found previously for models of capillary waves with dewetted islands $[39,40]$. The model holds for the early stages of dewetting, but it agrees quite well with the AFM PSD with larger polydispersity taken at the end of the experiment where there is already some start of the coarsening. This capillary wave contribution from the calculation is only expected at low in-plane wave vector $q_{\|}$, and a relaxation time can be calculated for the capillary waves ( $\left.\tau \sim 2.47 \times 10^{3} \mathrm{~s}[16,18,53]\right)$ for the bilayer film. At the peak of the PSD and beyond there are only contributions from the holes [16].

The total area covered by holes is $40.7 \%$ from the sampled area in Fig. 1(d), and the average size of the holes is $\sim 6500 \AA$. The footprint of the beam on the sample is given by $d / \sin \left(\theta_{\text {in }}\right)$; here, $d=20 \mu \mathrm{m}$ is the height on the incoming $\mathrm{x}$-ray beam, and $\theta_{\text {in }}=0.146^{\circ}$ is the incident grazing angle at the top surface. Thus the footprint of the beam on the sample is $7.85 \mathrm{~mm}$. From this, we estimate there to be $1.93 \times 10^{5}$ holes in the X-ray beam towards the end of the experiment. The large number of holes makes them a significant source of speckles in the coherent $\mathrm{x}$-ray beam.

The radiation damage from a bilayer sample was accessed by monitoring it at room temperature for the same number of CCD camera detector frames as used in the batches but with a much higher exposure time per frame. Both the static GISAXS intensity and autocorrelation functions from XPCS show no evidence of damage [16]. The GISAXS plot as a function of time and the measured autocorrelation functions using XPCS are included in the Supplemental Material in the section on radiation damage. Thus, at higher temperature by exposing the same sample area with less exposure time and moving to different parts of the sample in subsequent batches, the effects of beam damage are mitigated (see below).

\section{RESULTS AND DISCUSSION}

$\mathrm{X}$-ray image sequences of the evolution of average diffuse surface scattering during spinodal dewetting were taken in three consecutive batches. The duration of each batch was approximately $1000 \mathrm{~s}$. For each batch, translation to different parts of the sample was done to minimize x-ray damage. After a variable waiting time, denoted $t_{w}$, kinetic measurements were initiated. For the first batch after the quench, $t_{w}=978 \mathrm{~s}$; for the second batch, $t_{w}=1991 \mathrm{~s}$; and for the third batch, $t_{w}=3204 \mathrm{~s}$. The surface diffuse scattering [16] with the speckle averaged away is equivalent to a conven- tional GISAXS measurement and is shown in Figs. 2(a)-2(c). Scattering vectors with the same $q_{\|}$but different $q_{z}$ out of the plane were averaged together. This is appropriate as $q_{z} a \ll 1$, with $a$ being the amplitude of the spinodal fluctuations [54]. The time evolution of the speckle-averaged diffuse scattered intensity shows a well-defined peak whose intensity grows and shifts to a slightly lower in-plane wave vector $q_{\|}$with time [Fig. 2(a)]. For the diffuse scattered intensity in the second batch, a shoulder appears [indicated by an arrow in Fig. 2(b)]. This pronounced shoulder at wave vectors beyond the primary peak in $\left\langle I\left(q_{\|}\right)\right\rangle$, which later diminishes, could be linked to an increase in "structural polydispersity" [35-37] at intermediate times. The fact that the low- $q_{\|}$parts of the plots show an increase in Figs. 2(a)-2(c) means that unlike conventional phase separation there is no conservation law for the strain variable [55].

The theoretical PSD above fits well the first GISAXS scan of $\left\langle I\left(q_{\|}\right)\right\rangle$except at the largest $q_{\|}$[16]. Furthermore, the theoretical PSD fits well with the GISAXS at the end of the experiment (note that the PSD is not exactly proportional to $\left\langle I\left(q_{\|}\right)\right\rangle$except when $q_{z} \mathrm{C}\left(q_{\|}\right)$or $q_{z} a$ is small, where $C(q \|)$ is the PSD and $a$ is the amplitude of fluctuations [40]). In the $\left\langle I\left(q_{\|}\right)\right\rangle$at the lowest $q_{\|}$there is a very small capillary wave contribution [16]. In the $q_{\|}$range covered by $\left\langle I\left(q_{\|}\right)\right\rangle$the scattering is dominated by the hole's contribution at the peak and beyond.

The static average correlation function shows the scattering peak moving to the smaller $q_{\|}$with time, which reveals slow coarsening during early dewetting in analogy to bulk viscoelastic phase separation. Figures 2(d)-2(i) display $\log \left(q_{\| \max }\right)$ and $\log \left(\left\langle I\left(q_{\|}\right)\right\rangle_{\max }\right)$ versus $\log (t)$ suggesting two distinct stages of the spinodal dewetting. At the early times (first and second batches), $q_{\| \max }$ and $\left\langle I\left(q_{\|}\right)\right\rangle_{\max }$ vary slowly with time [10]. The power-law exponent of this coarsening process is considerably smaller than that for usual binary fluid phase separation [35-37] probably because the pattern evolution is dominated by the slow dynamics of the viscoelastic polymer matrix. At a later time, these surface structures coarsen with a larger exponent, showing a transient scaling law as seen in other viscoelastic phase-separated systems $\left(q_{\| \max } \sim t^{0.45}\right)[11,56]$. Recent simulations show that the mechanical relaxation of the dense phase plays a critical role for this unconventional power law but in the case of viscoelastic dewetting the intensity scaling with time is not linear as expected [57]. The peak intensity here grows strongly with time $\left(\sim t^{1.49}\right)$ as shown in Fig. 2(i). An additional consistency check on the analysis of the experimental data [7] is the expected relation $\beta=3 \alpha$, where $\alpha$ and $\beta$ are the time exponents defined by $q_{\| \max } \sim t^{-\alpha}$ and $\left\langle I\left(q_{\|}\right)\right\rangle_{\max } \sim t^{\beta}$, respectively. This means that the evolving length scale given by $q_{\| \max } \sim 1 / L$ remains almost statistically self-similar at later times. Here, $\left\langle I\left(q_{\|}\right)\right\rangle_{\max } \sim t^{1.49}$ grows as expected at late times, nearly consistent with $q_{\| \max } \sim t^{-0.45}$ [7].

The physics behind this transient power-law behavior in $q_{\| \max }$ versus time [Fig. 2(f)] is not clear at present. A stronger test of scaling is given by whether the scattering function itself scales. Here, there is imperfect self-similarity in the pattern evolution at scales both larger and smaller than the characteristic length scale $L$ as illustrated in Fig. 3(a). The scaled scattering function is not totally time independent, i.e., 

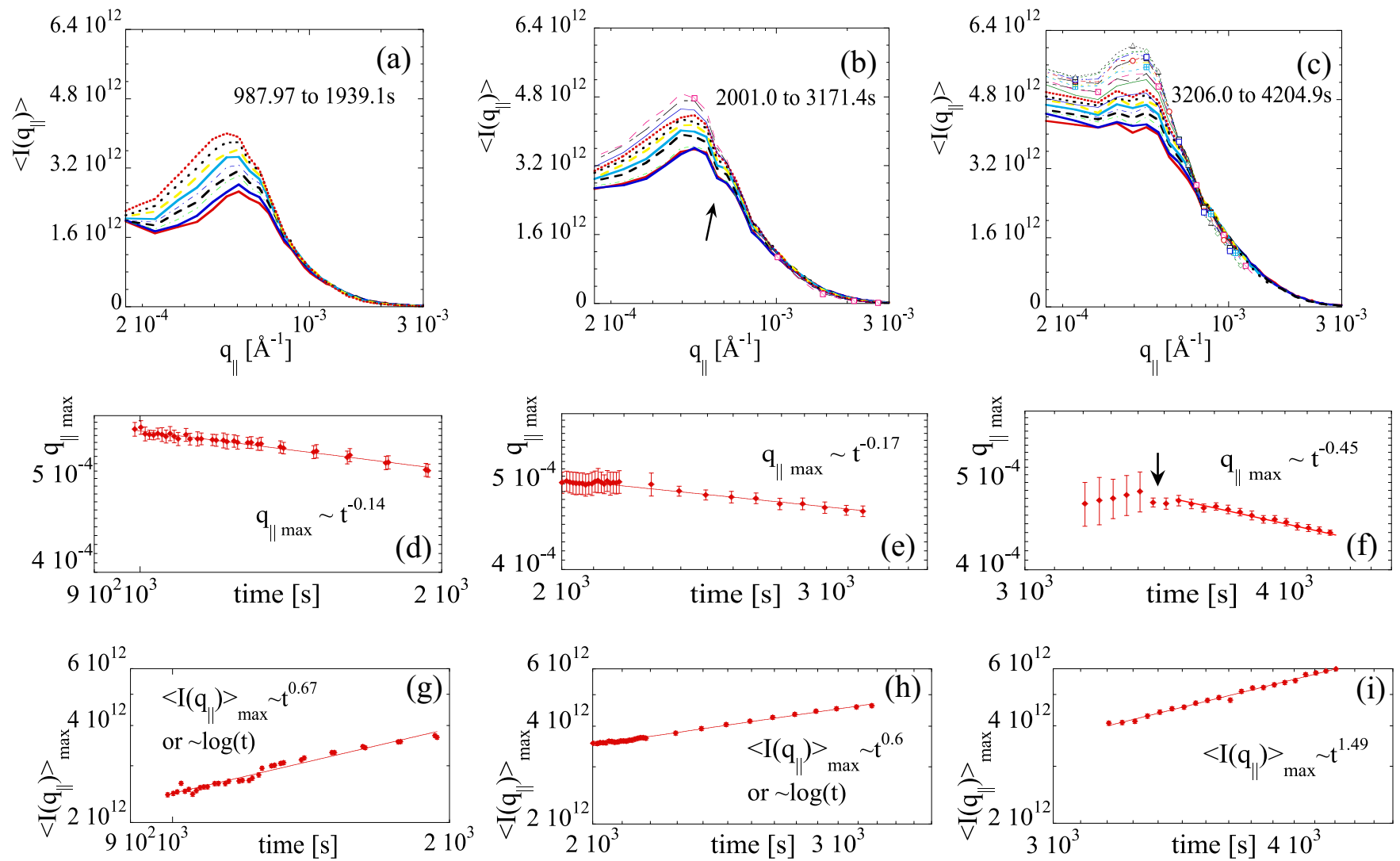

FIG. 2. Speckle-averaged surface diffuse scattering. (a)-(c) Time evolution of diffuse scattering intensity $\left\langle I\left(q_{\|}\right)\right\rangle$vs in-plane wave vector $q_{\|}$for three different batches at $165^{\circ} \mathrm{C}$, for the 180- $\AA$-thick top PBrS film (details in the Supplemental Material [16]). For the first batch, $t_{w}=978 \mathrm{~s}$ after the quench; for the second batch, $t_{w}=1991 \mathrm{~s}$; and for the third batch, $t_{w}=3204 \mathrm{~s}$. Through the different batches, the diffuse scattering records snapshots of the evolving top layer. (d)-(f) Log-log plots of the position of peak maximum $q_{\| \max }$ vs time showing a two-stage film breakup process: an almost stationary stage, followed in the end by intermediate coarsening behavior. The vertical error bars indicate errors in the determination of peak position from Gaussian fits after subtraction of background. (g)-(i) Plots of $\left\langle I\left(q_{\|}\right)\right\rangle_{\max }$ as a function of time. After the predominant period of slow growth, $\left\langle I\left(q_{\|}\right)\right\rangle_{\max }$ grows strongly. The vertical error bars indicate experimental errors.
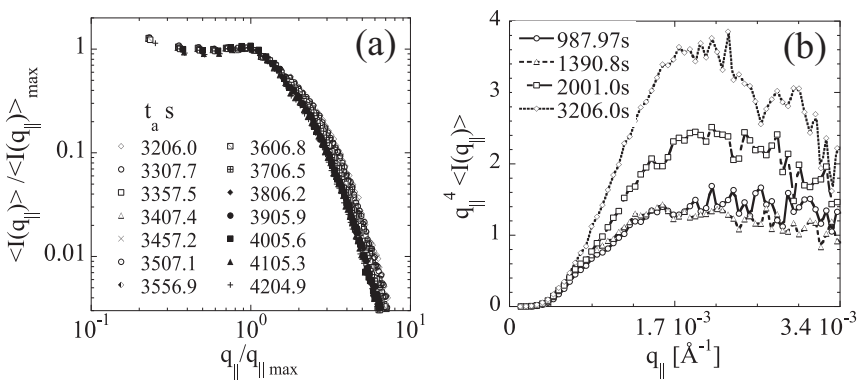

FIG. 3. (a) Scaling behavior of the average diffuse scattering structure factor $\left\langle I\left(q_{\|}\right)\right\rangle /\left\langle I\left(q_{\|}\right)\right\rangle_{\max }$ vs $q_{\|} / q_{\| \max }$ at different times after the start of the dewetting. The scaled scattering function at later times does not collapse onto a single master curve. This indicates that the self-similarity hypothesis and the dynamical scaling law, which are known to hold for fluid-fluid phase separation (model H), break down for viscoelastic phase separation as well as dewetting as shown here. (b) The $q_{\|}^{4}\left\langle I\left(q_{\|}\right)\right\rangle$vs $q_{\|}$plot (Kirste-Porod plot) for evolving time shows the augmentation of the peak position with time in quasi two dimensions. The peak in the plot appears when the scattering vector becomes of the same order of magnitude as the curvature of the interfaces, causing deviation from the asymptotic Porod's law. does not collapse onto a single curve (within error bars); specifically, the curves differ notably in the region just beyond the peak for the large- $q_{\|}$region [Fig. 3(a)] which defines smaller length scales, and it is a bit different at the very lowest $q$ due to constraints of the conservation law. This indicates that the self-similarity hypothesis and the dynamical scaling laws [7], which are known to hold for late-stage polymerpolymer dewetting (normal phase separation model $\mathrm{H}$; see Fig. 2(b) in Ref. [7]), do not hold for bulk viscoelastic phase separation [58] or for viscoelastic dewetting as shown here [Fig. 3(a)]. However, here the dynamic scaling does not work as the exponents $\alpha$ and $\beta$ depend on the time scales of the viscoelastic parameters, especially the relaxation time of the stress as shown below; thus it is then not so meaningful to discuss the value of the exponents. Porod's law is observed at large $q_{\|}$due to the presence of sharp well-defined interfaces. The asymptotic behavior of $q_{\|}$is less than Porod's exponent of 4 (starts at 3.77) until the middle of the second batch. This exponent steadily increases with time and is greater than Porod's exponent of 4. As the holes grow and become more circular, a Porod's exponent greater than 4 emerges due to curvature correction [59]. The $q_{\|}^{4}\left\langle I\left(q_{\|}\right)\right\rangle$plot (Kirste-Porod plot) for 

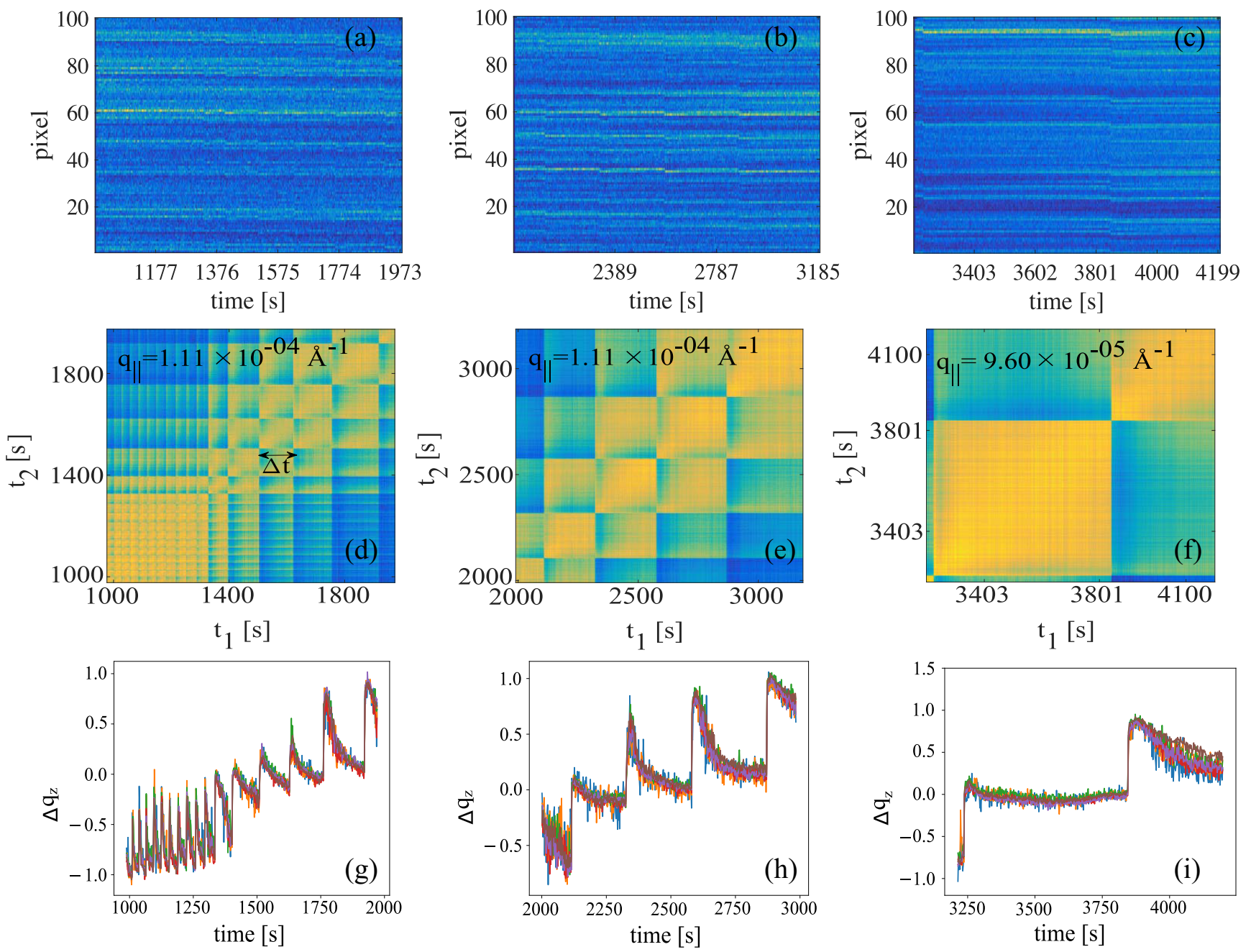

FIG. 4. Evolution of individual speckles and temporal and spatial correlation functions of the detailed speckle patterns [16]. (a)-(c) Waterfall plots showing the evolution of a 100-pixel cross section of a speckle pattern. Abrupt changes in the pixel position of the intensity indicate the occurrence of hole-initiation events in the top layer of the film. (d)-(f) The blocklike structure of TTCFs [Eq. (3)] reflects the intermittent nature of the dynamics in time. (a)-(f) use the PARULA color map from MATLAB; blue represents 0 on this scale. (g)-(i) Results of the spatial cross correlations [Eq. (4)] for different $|q|$ values for bins 5-10 (Supplemental Fig. S7(a) [16]). The $y$-axis units are pixels. $\Delta q_{z}$ per pixel is $2.1 \times 10^{-5} \AA^{-1}$. The different colors (blue, $0.0508 \AA^{-1}$; orange, $0.0487 \AA^{-1}$; green, $0.0467 \AA^{-1}$; red, $0.0446 \AA^{-1}$; purple, $0.0425 \AA^{-1}$; and brown, $0.0404 \AA^{-1}$ ) indicate the different $|q|$ shown in Supplemental Fig. S7(a) [16]. (g) Measured speckle shifts between each diffraction image and a reference image at $t=1563 \mathrm{~s}$ for batch 1 . (h) Measured speckle shifts between each diffraction image and a reference image at $t=2576 \mathrm{~s}$ for batch 2. (i) Measured speckle shifts between each diffraction image and a reference image at $t=3789 \mathrm{~s}$ for batch 3 .

evolving time shows the augmentation of the peak position with time [Fig. 3(b)]. The peak in the plot appears when the scattering vector becomes of the same order of magnitude as the curvature of the interfaces.

Figures 4(a)-4(c) show the time evolution of a 100-pixel cross section of a speckle pattern in the $q_{z}$ direction (see scattering geometry $[4,16])$ from surface diffuse scattering during dewetting, which is denoted a "waterfall" plot $[16,60]$. Typically, speckles disappear after a given relaxation time, but here something different is observed: speckle motion. Small shifts in the intensity of bright pixels show the speckle movement in real time. After each hole-opening event, the scattering pattern is seen to shift along the detector by a distance of the order of one pixel. This shift is caused by a stress-induced curvature of the silicon substrate supporting the sample which changes the outgoing scattering angle relative to the surface. The grazing-in-plane scattering geometry determines the mapping between the detector pixels and $q$ space. In this scattering geometry, curvature along the scattering plane (the $q_{z}$ direction) will lead to noticeable deflections of the scattered beam, while curvature transverse to the scattering direction will lead to negligible deflections of the beam due to the small grazing angle of incidence. For a sample movement in real space responsible for the speckle shifts, the outgoing angle from the sample is different; thus the shift is in total $|q| \sim\left|q_{z}\right|$ mostly as you cannot tell the difference between $|q|$ and $\left|q_{z}\right|$ as $q_{y}$ and $q_{x}$ are very small (Supplemental Fig. S7(a) [16]). The horizontal direction on the CCD camera falls along the $q_{x}$ and $q_{z}$ directions, and the vertical direction of the CCD camera falls along the $q_{y}$ direction. Pixels in the two-time correlation function below are binned along $q_{\|}=\sqrt{\left(q_{x}^{2}+q_{y}^{2}\right)}$, 
and $q_{z}$ dependence is ignored since $q_{z} a \ll 1$, with $a$ being the amplitude of surface height fluctuations. The direction of the speckle shifts is horizontal on the detector along the negative direction in $q_{z}$ (or equivalently $q_{x}$ ), i.e., towards the beam center [Figs. 4(a)-4(c)] [16]. Furthermore, there is no speckle movement at the same time in the waterfall plots in the $q_{y}$ direction [16].

Speckle correlations were initially analyzed using the twotime correlation function (TTCF) as defined in earlier work [4]:

$$
C\left(q_{\|}, t_{1}, t_{2}\right)=\frac{\left\langle I\left(q_{\|}, t_{1}\right) I\left(q_{\|}, t_{2}\right)\right\rangle}{\left\langle I\left(q_{\|}, t_{1}\right)\right\rangle\left\langle I\left(q_{\|}, t_{2}\right)\right\rangle} .
$$

Here, $\langle\cdots\rangle$ denotes averaging over pixels at equivalent wave vectors. The TTCF measures how the structure on a given length scale changes, i.e., the statistical similarity of intensity between time $t_{1}$ and time $t_{2}$ as the sample evolves. The TTCF shows a decorrelation of intensity fluctuations described by a steplike function (shown as sharp edges of the blocks, where the correlation amplitude changes rapidly; the color scale shows the degree of correlation) corresponding to fast elastic deformation due to new hole initiations. The signal jumps and goes back [Figs. 4(d)-4(f)]. The iteration of many holeinitiation and hole-growth events leading to the fast elastic polymer deformation described above can be observed as discrete decorrelations in the TTCFs. The TTCFs are plotted in Figs. 4(d) -4 (f) for $q_{\|} \sim 1.0 \times 10^{-4} \AA^{-1}$. Blocks in the TTCF [Figs. 4(d)-4(f)] correspond to time durations of minimal variation in the speckle intensity configuration and correspond to normal relaxation of the deformation (coarsening) polymer matrix. The decorrelations observed in the TTCF are slow enough to be observable in a viscoelastic top thin film, unlike the case of fluid phase separation, where once started, these pinch-off (hole-initiation) events occur too rapidly as not to impede the subsequent coarsening process [35-37]. In this "punctuated viscoelastic spinodal dewetting" rather than a normal spinodal dewetting, more hole-initiation events happen within a narrow time interval at the very beginning [batch 1, Fig. 4(d)]. At later times the frequency of hole opening decreases with the onset of coarsening holes at late time [Fig. 4(f)].

The initial response due to the hole-initiation event in the film is elastic (sharp jumps [Figs. 4(d)-4(f)]), with time increasing duration of viscous relaxation processes, i.e., growth of holes is observed. Such behavior of rapid hole formation (rupture) and relaxation or hole growth (coarsening) is repeated with decreasing frequency until all the stresses are relieved due to rupture. Finally, mostly hole growth or coarsening driven by surface tension takes over [Figs. 1(c) and 2(f)]. The early-time behavior of TTCF is wave vector $q_{\|}$ independent (first and second batches) and shows slight $q_{\|}$dependence at the late times when coarsening starts to increase (third batch) [16].

The TTCFs here do not represent standard XPCS correlation functions but require an understanding of the individual decorrelations related to speckle shifts seen in the dewetting kinetics. This requires going beyond the standard TTCF analysis. Furthermore, the ability to measure small deformations due to small sample curvature causing pixel shifts on the detector using coherent $\mathrm{x}$ rays is useful for understanding many

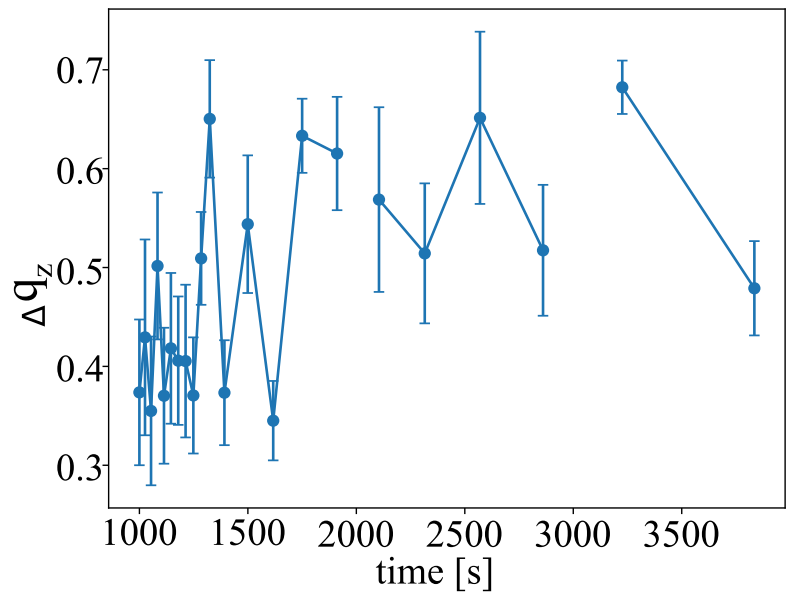

FIG. 5. The $y$-axis units are pixels. $\Delta q_{z}$ per pixel is $2.1 \times 10^{-5}$ $\AA^{-1}$. The jumps shown in Figs. 4(g)-4(i) averaged over $|q|$ values as shown by the $11|q|$ regions in the average image of the diffuse scattering on the CCD (Supplemental Fig. S7(a) [16]), or the mean calculated from the data (Supplemental Fig. S8(a) [16]). The three batches are plotted together. The solid line connects the average shifts at the jumps in each individual batch.

aspects of disordered materials as shown below [61]. In order to track speckle motion, a cross-correlation function, Eq. (4), is introduced which tracks correlations at time $t_{1}$ of speckles at wave vector $q$ with speckles centered at wave vector $q+\Delta q$ at time $t_{2}$ defined by [61] (Supplemental Fig. S7(a) [16])

$$
C C\left(q_{0}, \Delta q, t_{1}, t_{2}\right)=\frac{\left\langle I\left(q, t_{1}\right) I\left(q+\Delta q, t_{2}\right)\right\rangle_{q_{0}}}{\left\langle I\left(q, t_{1}\right)\right\rangle_{q_{0}}\left\langle I\left(q+\Delta q, t_{2}\right)\right\rangle_{q_{0}}} .
$$

Here, \langle\rangle$_{q_{0}}$ means averaging over a small region of $q$ around $q_{0}$. The results of such cross correlations are shown in Figs. 4(g)-4(i); the speckle shifts (in pixel units) are calculated in the negative $z$ direction, where they are the most significant as a function of $|q|$ and time. The jumps in the shifts correspond to the decorrelations seen in the measured TTCFs. These spatial shifts are independent of $|q|$, except at late times beyond $3750 \mathrm{~s}$ (Figs. 4(g)-4(i) and Supplemental Fig. S8(a) [16]).

Figure 5 shows the size of jumps as determined from cross-correlating frame $i$ to $i+1$ averaged over $|q|$ values as shown by the $11|q|$ regions in the average image of the diffuse scattering on the CCD for all three batches (Supplemental Figs. S7 (a) and S8(a) [16]). Here, the cross-correlation analysis allows for comparison of the speckle positions before the jump with the positions after the jump, not with the top of the maximum shift shown in Figs. 4(g)-4(i). The large shift at the jump independent of $|q|$ corresponds to a slight change in the curvature of the silicon wafer [16]. The stress that the film induces (at short times) in the substrate causes a slight curvature change of the order of a pixel or less on the detector. The relationship between the stress in a thin film and the resulting substrate curvature is given by the Stoney formula [62]. This can then be used to convert these average shifts at the jumps to estimates of average stress in the top polymer layer $[16,63,64]$. The values of stress at jumps are approximately $0.1 \mathrm{GPa}$ in the beginning and vary slowly with each new jump increase up to $0.2 \mathrm{GPa}$. In order for the film to 
sustain this stress, the stress must be smaller than the Young's modulus of the film, which is, indeed, a factor of nearly 10 times larger, $1.5-1.7 \mathrm{GPa}[65,66]$.

The initial response to hole formation is solidlike due to the large elastic energy stored in the connected film structure. This starts as an inhomogeneous stress distribution, leading to the existence of spatial correlation in the stress field, as indicated by features such as the array of holes formed [Fig. 1(d)] [38]. The initially nonuniform distribution of stress first becomes uniform through elastic processes and then, over a longer time, dissipates due to viscous relaxation.

The duration of the jumps themselves is short; they show up between two frames. Furthermore, in Figs. 4(g)-4(i) one observes that after a jump (largest shift or stress) the shifts decay with a time constant $\tau$. After $1400 \mathrm{~s}, \tau$ seems to get increasingly longer. The relaxation of the stress after each jump is fitted to an exponential decay. The exact relaxation time is somewhat uncertain from these fits as the stress does not fully relax before the next event occurs, leading to an uncertainty in the amplitude of the exponential fit. Nevertheless, the exponential function gives a fitted time constant of the decay [16]. The time constants $\tau$ before $1400 \mathrm{~s}$ in Fig. 4(g) are less than the reptation time of $33 \mathrm{~s}[16,18,67]$; hence the response is elastic or solidlike (i.e., the deformation is instantaneous, small, and almost reversible; this is when holes likely form in arrays as shown by AFM). After $1400 \mathrm{~s}, \tau$ gets longer, and the response is viscoelasticlike (instantaneous elastic deformation followed by viscous time-dependent deformation). After $1400 \mathrm{~s}, \tau$ is larger than the reptation time at certain jumps and gets generally longer, which is the beginning of viscouslike behavior and the start of diffusive coarsening during dewetting. In fact, the diffusion process must accompany the contraction of a more elastic polymer phase to avoid the inhomogeneous deformation of the still-connected polymer film. Recent simulations [57] show that the mechanical rupture (in analogy here, the hole opening at the jump) is a rapid nonlinear process; it is not the limiting process controlling the coarsening due to hole growth. It is the slow mechanical relaxation of the polymer film that controls the coarsening. The whole polymer film relaxes its shape while retaining the mechanical balance condition [57]. Change in the polymer film structure and the resulting slow mechanical relaxation are the primary mechanisms of the late coarsening observed here. Thus spatial cross correlation can track speckles in time and provide information on how the polymer film responds to stress due to hole-initiation events and hole growth.

In addition to the abrupt decorrelation of the two-time correlation function, there is also a more gradual change in the contrast of the speckle pattern as the frame-to-frame speckle offset slowly shifts back towards zero. This can be explained as follows: The initial jump is due to curvature of the substrate, which shifts the speckles but initially only results in a small area change. As time progresses, the holes grow, reducing the total area (and contrast). Each cross correlation $[61,68]$ has a peak amplitude of $1+\beta$ ( $\beta$ is the speckle contrast) sitting on a background of height 1 . The cross-correlation analysis shows that not only the jumps but also all shifts are essentially independent of $|q|$ [Figs. 4(g)-4(i)] but there is a slight decrease in the contrast $\beta$ for shifts but not for the
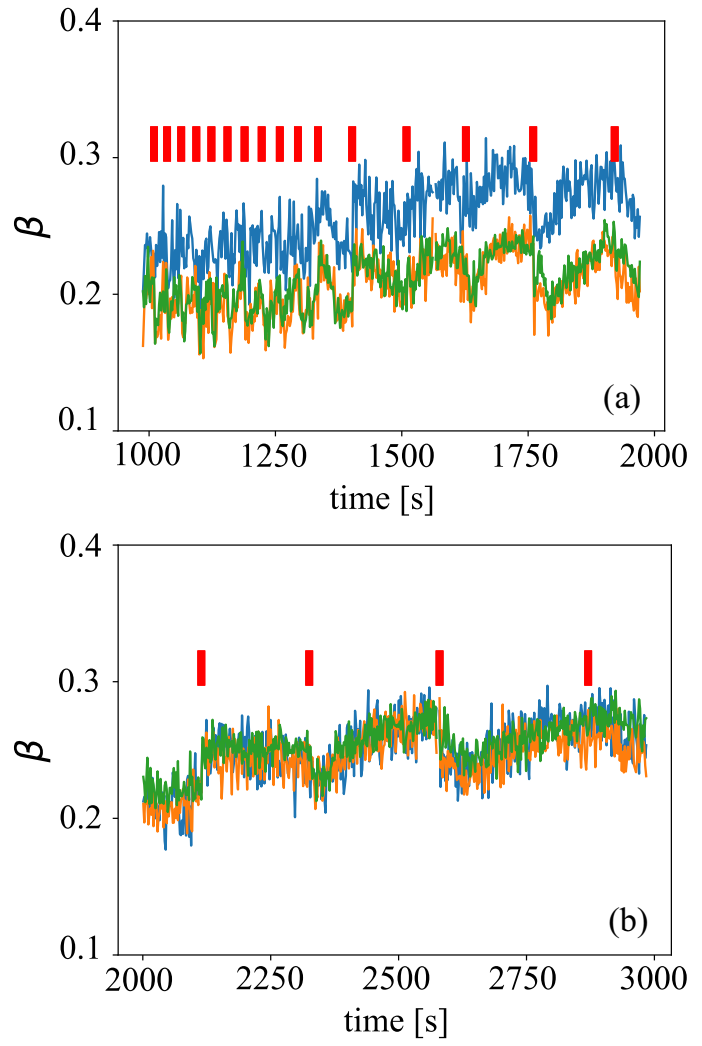

FIG. 6. Plots of contrast $\beta$ vs time for (a) batch 1 and (b) batch 2 are shown. The $\beta$ for $|q|$ 's $0.0446 \AA^{-1}$ (blue, bin 8), $0.0425 \AA^{-1}$ (orange, bin 9), and $0.0404 \AA^{-1}$ (green, bin 10) are plotted (Supplemental Fig. S7(a) [16]). The centers of the red bars are at the times of the jump.

jumps [Figs. 6(a) and 6(b)]. This reduction in the contrast is interpreted as being due to the reduction of the overall sample area as a result of the opening of holes and growth. Specifically, if the total area $A$ is reduced by $\Delta A$, then the contrast will be reduced by a factor of $1-\Delta A / A$. The total area change then is estimated due to hole opening from this factor. Using this, $\Delta A / A$ is measured varying between 1 and $8 \%$ close to jumps [16]. This fractional area change from the contrast change is also consistent with the observed stress, which should be given by $G \Delta A / A(0.1-0.2 \mathrm{GPa})$, where $G$ is the Young's modulus of the thin polymer film. Furthermore, this is consistent with the total area covered by holes $(40.7 \%$ observed by AFM) and 22 events observed in Figs. 4(g)-4(i) corresponding to a $2 \%$ change in surface area per event.

Figure 7 shows that the time $\Delta t$ between the decorrelating jumps or events increases as the "dewetting time" $t$ [Figs. 4(d)-4(i)] with remarkable regularity. Furthermore, coarsening behavior is observed over increasing time intervals as the system evolves [Figs. 4(d)-4(i)]. In Fig. 7 the holeinitiation event duration rate (causing jumps), $1 / \Delta t$, is plotted versus time $t$. A well-defined power law is observed: $1 / \Delta t$ decreases as $1 / t^{3 / 2}$ with time (Fig. 7). In the case of dewetting the statistical behavior of this power law is only an observation that currently cannot be modeled to a specific physical mechanism. However, the $1 / t$ decrease in rate is observed in a wide 


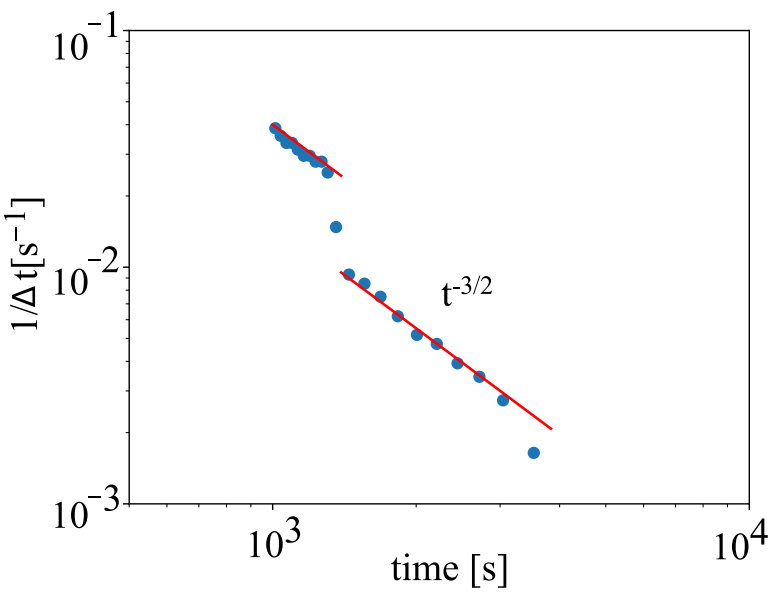

FIG. 7. The hole-initiation event duration rate (time $\Delta t$ is the time between decorrelating events or jumps) decreases with time as $1 / t^{3 / 2}$.

range of systems where stress is periodically released, such as earthquake aftershocks, and is known as the Omori law [60] or Omori-Utsu law where power laws with an exponent $p$ with a wider range of values between 0.9 and 1.5 are observed $[69,70]$. These are empirical laws, though recent simulations show the dependence of the decay exponent $p$ on the power exponent $n$ of the nonlinear viscoelasticity [69-71].

\section{CONCLUSIONS}

In viscoelastic dewetting, coarsening is driven mainly by the mechanical force in the hole-initiation stage, whereas it is driven by the thermodynamic force in the final stage. In the final stage, the elastic deformation of the polymer is less frequent, which leads to the weakening of the resulting stress fields. Furthermore, in analogy to viscoelastic phase separation, the absence of ideal self-similarity is obvious in the scaled structure factor [Fig. 3(a)]. The PBrS polymer matrix eventually behaves in a more viscouslike manner, and the domain shape is of the lowest interfacial energy (circular) as is usual in normal dewetting [Fig. 1(d)].

A system that has not yet fully relaxed to equilibrium retains memories of its creation, while one that is in equilibrium has no memory of its past [72]. There is memory built in during dewetting hole formations due to anisotropic stress distribution within the viscoelastic film (recent movement of polymer molecules due to hole formations determines what happens next), and the anisotropic stress distribution is correlated to the next sequence of events, i.e., hole formation in arrays. This process is clearly shown by the AFM image taken at the end of the process, which shows hole formation in one-dimensional arrays due to elastic stress.

TTCFs reveal the kinetics of elastic deformation due to hole formation and growth and stress relaxation during growth as seen through the checkerboardlike pattern [Figs. 4(d)-4(f)]. Here, each new hole-formation event leads to a decorrelation or jump and a resulting discontinuity in the TTCF. The timing of these jump events is very regular indicating that each hole-formation event depends on the elapsed time since the previous event, supporting the idea of stress relaxation.
This simple model of stress relaxation does not explain, however, why the time spacing between events increases with the overall time from the initial quench, as seen in the observed power-law dependence (Fig. 7). Assume that the area created by a hole-opening event is almost a constant. Then, if the total film area decreases with time, the fractional change in area will increase with each hole-opening event, and consequently, the elastic energy associated with each new opening event is larger than the elastic energy associated with the previous one. This implies that it takes a longer time between events for the strain or deformation to relax as time progresses. This effect will also be strongly influenced by the transition from hole opening to hole growth, as the growth of holes also reduces the total area.

The kinetics are best explained by drawing an analogy to viscoelastic phase separation [9]. Here, speckles give us sensitivity to periodic stress changes in the film which curves the substrate. Rather than just switching from a stress-field-based order parameter to a surface-height-based (composition-based) order parameter as seen in bulk viscoelastic phase separation, a series of cascades is found, where it is conjectured that the order parameter switches back and forth from stress to surface height with increasingly long periods between switching. This order parameter switching slows down in time, following a pattern typical of systems relieving stress, known as the Omori-Utsu law. This stress relief power-law behavior is observed here in thin dewetting polymer films. This behavior has important implications for our understanding of viscoelastic dewetting and viscoelastic phase separation.

Dynamic viscoelasticity comes into play during this kinetic process of spinodal dewetting [8-11]. Normally, the phenomenological constitutive relation crucially depends on the composition dependence of the elastic moduli and the mechanical relaxation times in the nonequilibrium process of dewetting. However, there is no firm basis for the physical description of these quantities. For a more quantitative understanding of viscoelastic phase separation and dewetting it is crucial to overcome this challenge. The coherent $\mathrm{x}$-ray techniques used in this experiment can reveal the viscoelastic kinetics which are otherwise hidden in the average scattering using incoherent $\mathrm{x}$ rays. Using the coherent speckle evolution gives the unique ability to measure stress and its relaxation in situ in the top dewetting film.

All data needed to evaluate the conclusions in this paper are present in this paper and/or the Supplemental Material [16]. Additional data are available from the authors upon request.

\section{ACKNOWLEDGMENTS}

This work was supported by the U.S. Department of Energy, Office of Science, Office of Basic Energy Sciences. This research used resources of the Advanced Photon Source and the Center for Nanoscale Materials, US Department of Energy (DOE) Office of Science User Facilities operated for the DOE Office of Science by Argonne National Laboratory under Contract No. DE-AC02-06CH11357. We also acknowledge Dr. T. Kawaguchi for his help with the schematic illustration. 
[1] G. Reiter, Dewetting of Highly Elastic Thin Polymer Films, Phys. Rev. Lett. 87, 186101 (2001).

[2] K. Dalnoki-Veress, B. G. Nickel, C. Roth, and J. R. Dutcher, Hole formation and growth in freely standing polystyrene films, Phys. Rev. E 59, 2153 (1999).

[3] H. Bodiguel and C. Fretigny, Viscoelastic dewetting of a polymer film on a liquid substrate, Eur. Phys. J. E: Soft Matter Biol. Phys. 19, 185 (2006).

[4] J. Lal, S. Malkova, M. K. Mukhopadhyay, S. Narayanan, A. Fluerasu, S. B. Darling, L. B. Lurio, and M. Sutton, Dewetting in immiscible polymer bilayer films, Phys. Rev. Mater. 1, 015601 (2017).

[5] F. Saulnier, E. Raphaël, and P.-G. de Gennes, Dewetting of Thin Polymer Films near the Glass Transition, Phys. Rev. Lett. 88, 196101 (2002).

[6] F. Saulnier, E. Raphaël, and P.-G. de Gennes, Dewetting of thinfilm polymers, Phys. Rev. E 66, 061607 (2002).

[7] J. Lal, L. B. Lurio, D. Liang, S. Narayanan, S. B. Darling, and M. Sutton, Universal dynamics of coarsening during polymerpolymer thin-film spinodal dewetting kinetics, Phys. Rev. E 102, 032802 (2020).

[8] H. Tanaka, Universality of Viscoelastic Phase Separation in Dynamically Asymmetric Fluid Mixtures, Phys. Rev. Lett. 76, 787 (1996)

[9] H. Tanaka, Viscoelastic model of phase separation, Phys. Rev. E 56, 4451 (1997).

[10] H. Tanaka, Unusual Phase Separation in a Polymer Solution Caused by Asymmetric Molecular Dynamics, Phys. Rev. Lett. 71, 3158 (1993).

[11] H. Tanaka and Y. Nishikawa, Viscoelastic Phase Separation of Protein Solutions, Phys. Rev. Lett. 95, 078103 (2005).

[12] G. Reiter, Dewetting of Thin Polymer Films, Phys. Rev. Lett. 68, 75 (1992).

[13] G. Reiter, Unstable thin polymer films: Rupture and dewetting processes, Langmuir 9, 1344 (1993).

[14] F. Brochard Wyart, P. Martin, and C. Redon, Liquid/liquid dewetting, Langmuir 9, 3682 (1993).

[15] M. Sferrazza, M. Heppenstall-Butler, R. Cubitt, D. Bucknall, J. Webster, and R. A. L. Jones, Interfacial Instability Driven by Dispersive Forces: The Early Stages of Spinodal Dewetting of a Thin Polymer Film on a Polymer Substrate, Phys. Rev. Lett. 81, 5173 (1998).

[16] See Supplemental Material at http://link.aps.org/supplemental/ 10.1103/PhysRevResearch.3.043162 for details.

[17] X. Hu, X. Jiao, S. Narayanan, Z. Jiang, S. Sinha, L. B. Lurio, and J. Lal, Resonantly enhanced off-specular X-ray scattering from polymer/polymer interfaces, Eur. Phys. J. E: Soft Matter Biol. Phys. 17, 353 (2005).

[18] X. Hu, Z. Jiang, S. Narayanan, X. Jiao, A. Sandy, S. K. Sinha, L. B. Lurio, and J. Lal, Observation of a low-viscosity interface between immiscible polymer layers, Phys. Rev. E 74, 010602(R) (2006).

[19] F. Ziebert and E. Raphaël, Dewetting of thin polymer films: Influence of interface evolution, EPL (Europhys. Lett.) 86, 46001 (2009).

[20] J. van Meerveld, A method to extract the monomer friction coefficient from the linear viscoelastic behavior of linear, entangled polymer melts, Rheol. Acta 43, 615 (2004).

[21] A. M. Higgins, M. Sferrazza, R. A. L. Jones, P. C. Jukes, J. S. Sharp, L. E. Dryden, and J. Webster, The timescale of spinodal dewetting at a polymer/polymer interface, Eur. Phys. J. E: Soft Matter Biol. Phys. 8, 137 (2002).

[22] R. Seemann, S. Herminghaus, and K. Jacobs, Dewetting Patterns and Molecular Forces: A Reconciliation, Phys. Rev. Lett. 86, 5534 (2001).

[23] J. P. de Silva, M. Geoghegan, A. M. Higgins, G. Krausch, M.-O. David, and G. Reiter, Switching Layer Stability in a Polymer Bilayer by Thickness Variation, Phys. Rev. Lett. 98, 267802 (2007).

[24] J. N. Israelachvili, Intermolecular and Surface Forces, 2nd ed. (Academic, London, 1991).

[25] M. Sferrazza, C. Xiao, R. A. L. Jones, D. G. Bucknall, J. Webster, and J. Penfold, Evidence for Capillary Waves at Immiscible Polymer/Polymer Interfaces, Phys. Rev. Lett. 78, 3693 (1997).

[26] M. C. Martínez, S. H. López, and E. V. Santiago, Relationship between polymer dielectric constant and percolation threshold in conductive poly(styrene)-type polymer and carbon black composites, J. Nanomater. 2015, 607896 (2015).

[27] A. Vrij, Possible mechanism for the spontaneous rupture of thin, free liquid films, Discuss. Faraday Soc. 42, 23 (1966).

[28] M. Rauscher, R. Blossey, A. Münch, and B. Wagner, Spinodal dewetting of thin films with large interfacial slip: Implications from the dispersion relation, Langmuir 24, 12290 (2008).

[29] W. W. Zhang and J. R. Lister, Similarity solutions for van der Waals rupture of a thin film on a solid substrate, Phys. Fluids 11, 2454 (1999).

[30] D. Vaynblat, J. R. Lister, and T. P. Witelski, Rupture of thin viscous films by van der Waals forces: Evolution and selfsimilarity, Phys. Fluids 13, 1130 (2001).

[31] D. Peschka and B. Niethammer, Thin-film rupture for large slip, J. Eng. Math. 66, 33 (2010).

[32] S. S. Thete, C. Anthony, O. A. Basaran, and P. Doshi, Selfsimilar rupture of thin free films of power-law fluids, Phys. Rev. E 92, 023014 (2015).

[33] V. Garg, P. M. Kamat, C. R. Anthony, S. S. Thete, and O. A. Basaran, Self-similar rupture of thin films of power-law fluids on a substrate, J. Fluid Mech. 826, 455 (2017).

[34] J. W. Cahn, Phase separation by spinodal decomposition in isotropic systems, J. Chem. Phys. 42, 93 (1965).

[35] S. I. Jury, P. Bladon, S. Krishna, and M. E. Cates, Tests of dynamical scaling in three-dimensional spinodal decomposition, Phys. Rev. E 59, R2535 (1999).

[36] V. Kendon, M. E. Cates, I. Pagonabarraga, J.-C. Desplat, and P. Bladon, Inertial effects in three-dimensional spinodal decomposition of a symmetric binary fluid mixture: A lattice Boltzmann study, J. Fluid Mech. 440, 147 (2001).

[37] I. Pagonabarraga, J.-C. Desplat, A. J. Wagner, and M. E. Cates, Interfacial dynamics in 3D binary fluid demixing: animation studies, New J. Phys. 3, 9 (2001).

[38] T. Araki and H. Tanaka, Simple tools for complex phenomena: Viscoelastic phase separation captured by disconnectable springs, Phys. Rev. E 72, 041509 (2005).

[39] M. Tolan, O. H. Seeck, J.-P. Schlomka, W. Press, J. Wang, S. K. Sinha, Z. Li, M. H. Rafailovich, and J. Sokolov, Evidence for Capillary Waves on Dewetted Polymer Film Surfaces: A Combined X-ray and Atomic Force Microscopy Study, Phys. Rev. Lett. 81, 2731 (1998). 
[40] M. Tolan, X-Ray Scattering from Soft-Matter Thin Films, Springer Tracts in Modern Physics Vol. 148 (Springer-Verlag, Berlin, 1999).

[41] H. Tanaka, Phase separation in soft matter: The concept of dynamic asymmetry, in Soft Interfaces, edited by L. Bocquet, D. Quéré, T. A. Witten, and L. F. Cugliandolo (Oxford University Press, Oxford, 2017), Chap. 15, pp. 465-521.

[42] N. Toyoda, M. Takenaka, S. Saito, and T. Hashimoto, Experimental studies of stress-diffusion coupling in semi-dilute polymer solutions. I. 'Viscoelastic length' and viscoelastic effects on early stage spinodal decomposition, Polymer 42, 9193 (2001).

[43] M. Takenaka, H. Takeno, T. Hashimoto, and M. Nagao, Viscoelastic effects on early stage of spinodal decomposition in dynamically asymmetric polymer blends, J. Chem. Phys. 124, 104904 (2006).

[44] H. Tanaka and T. Araki, Phase Inversion during Viscoelastic Phase Separation: Roles of Bulk and Shear Relaxation Moduli, Phys. Rev. Lett. 78, 4966 (1997).

[45] G. Debrégeas, P. Martin, and F. Brochard-Wyart, Viscous Bursting of Suspended Films, Phys. Rev. Lett. 75, 3886 (1995).

[46] F. Brochard-Wyart, G. Debregeas, R. Fondecave, and P. Martin, Dewetting of supported viscoelastic polymer films: Birth of rims, Macromolecules 30, 1211 (1997).

[47] D. Slep, J. Asselta, M. H. Rafailovich, J. Sokolov, D. A. Winesett, A. P. Smith, H. Ade, and S. Anders, Effect of an interactive surface on the equilibrium contact angles in bilayer polymer films, Langmuir 16, 2369 (2000).

[48] H. Tanaka and T. Araki, Spontaneous coarsening of a colloidal network driven by self-generated mechanical stress, EPL (Europhys. Lett.) 79, 58003 (2007).

[49] H. Tanaka, Phase-separation kinetics in dynamically asymmetric binary fluids: Viscoelastic effects in polymer solutions, Int. J. Thermophys. 16, 371 (1995).

[50] M. Sutton, A review of x-ray intensity fluctuation spectroscopy, C. R. Phys. 9, 657 (2008).

[51] S. Huettenbach, M. Stamm, G. Reiter, and M. Foster, The interface between two strongly incompatible polymers: Interfacial broadening and roughening near $T_{g}$, Langmuir 7, 2438 (1991).

[52] P. Müller-Buschbaum and M. Stamm, Correlated roughness, long-range correlations, and dewetting of thin polymer films, Macromolecules 31, 3686 (1998).

[53] Z. Jiang, H. Kim, S. G. J. Mochrie, L. B. Lurio, and S. K. Sinha, Surface and interfacial dynamics of polymeric bilayer films, Phys. Rev. E 74, 011603 (2006).

[54] S. K. Sinha, E. B. Sirota, S. Garoff, and H. B. Stanley, X-ray and neutron scattering from rough surfaces, Phys. Rev. B 38, 2297 (1988).

[55] G. B. Stephenson, Spinodal decomposition in amorphous systems, J. Non-Cryst. Solids 66, 393 (1984).
[56] J. Lal and R. Bansil, Light-scattering study of kinetics of spinodal decomposition in a polymer solution, Macromolecules 24, 290 (1991)

[57] M. Tateno and H. Tanaka, Power-law coarsening in networkforming phase separation governed by mechanical relaxation, Nat. Commun. 12, 912 (2021).

[58] H. Tanaka and T. Araki, Viscoelastic phase separation in soft matter: Numerical-simulation study on its physical mechanism, Chem. Eng. Sci. 61, 2108 (2006).

[59] R. Kirste and G. Porod, Röntgenkleinwinkelstreuung an kolloiden Systemen. Asymptotisches Verhalten der Streukurven, Kolloid Z. 184, 1 (1962).

[60] C. Sanborn, K. F. Ludwig, M. C. Rogers, and M. Sutton, Direct Measurement of Microstructural Avalanches during the Martensitic Transition of Cobalt Using Coherent X-ray Scattering, Phys. Rev. Lett. 107, 015702 (2011).

[61] M. Sutton, J. R. M. Lhermitte, F. Livet, and F. Ehrburger-Dolle, High resolution strain measurements in highly disordered materials, Phys. Rev. Res. 3, 013119 (2021).

[62] G. G. Stoney, The tension of metallic films deposited by electrolysis, Proc. R. Soc. London, Ser. A 82, 172 (1909).

[63] W. D. Nix, Mechanical properties of thin films, Metall. Trans. A 20, 2217 (1989).

[64] M. R. Ardigo, M. Ahmed, and A. Besnard, Stoney formula: Investigation of curvature measurements by optical profilometer, Adv. Mater. Res. 996, 361 (2014).

[65] Y. Liu, Y.-C. Chen, S. Hutchens, J. Lawrence, T. Emrick, and A. J. Crosby, Directly measuring the complete stress-strain response of ultrathin polymer films, Macromolecules 48, 6534 (2015).

[66] C. M. Stafford, B. D. Vogt, C. Harrison, D. Julthongpiput, and R. Huang, Elastic moduli of ultrathin amorphous polymer films, Macromolecules 39, 5095 (2006).

[67] J. T. Seitz, The estimation of mechanical properties of polymers from molecular structure, J. Appl. Polym. Sci. 49, 1331 (1993).

[68] J. R. M. Lhermitte, M. C. Rogers, S. Manet, and M. Sutton, Velocity measurement by coherent x-ray heterodyning, Rev. Sci. Instrum. 88, 015112 (2017).

[69] E. A. Jagla, Aftershock production rate of driven viscoelastic interfaces, Phys. Rev. E 90, 042129 (2014).

[70] H. Sakaguchi and K. Okamura, Aftershocks and Omori's law in a modified Carlson-Langer model with nonlinear viscoelasticity, Phys. Rev. E 91, 052914 (2015).

[71] X. Zhang and R. Shcherbakov, Power-law rheology controls aftershock triggering and decay, Sci. Rep. 6, 36668 (2016).

[72] N. C. Keim, J. D. Paulsen, Z. Zeravcic, S. Sastry, and S. R. Nagel, Memory formation in matter, Rev. Mod. Phys. 91, 035002 (2019).

Correction: The omission of a funding statement in the Acknowledgment section has been fixed. 